\title{
Limits for the Firehose Instability in Space Plasmas
}

\author{
M. Lazar · S. Poedts
}

Received: 14 April 2009 / Accepted: 17 June 2009 / Published online: 10 July 2009

(C) Springer Science+Business Media B.V. 2009

\begin{abstract}
Electromagnetic instabilities in high- $\beta$ plasmas, where $\beta$ is the ratio of the kinetic plasma energy to the magnetic energy, have a broad range of astrophysical applications. The presence of temperature anisotropies $T_{\|} / T_{\perp}>1$ (where $\|$ and $\perp$ denote directions relative to the background magnetic field) in solar flares and the solar wind is sustained by the observations and robust acceleration mechanisms that heat plasma particles in the parallel direction. The surplus of parallel kinetic energy can excite either the Weibel-like instability (WI) of the ordinary mode perpendicular to the magnetic field or the firehose instability (FHI) of the circularly polarized waves at parallel propagation. The interplay of these two instabilities is examined. The growth rates and the thresholds provided by the kinetic Vlasov-Maxwell theory are compared. The WI is the fastest growing one with a growth rate that is several orders of magnitude larger than that of the FHI. These instabilities are however inhibited by the ambient magnetic field by introducing a temperature anisotropy threshold. The WI admits a larger anisotropy threshold, so that, under this threshold, the FHI remains the principal mechanism of relaxation. The criteria provided here by describing the interplay of the WI and FHI are relevant for the existence of these two instabilities in any space plasma system characterized by an excess of parallel kinetic energy.
\end{abstract}

Keywords Corona $\cdot$ Flares, dynamics $\cdot$ Solar wind $\cdot$ Instabilities $\cdot$ Waves, plasma

\section{Introduction}

The origin of the particle-velocity anisotropies and their relaxation mechanisms in space plasmas have been extensively investigated during the past few decades. Interplanetary plasmas are magnetized and low or noncollisional. As a result, the electrons and ions can easily develop heat-flux and temperature anisotropies, and their velocity distribution

M. Lazar $(\bowtie) \cdot$ S. Poedts

Center for Plasma Astrophysics, K.U. Leuven, Celestijnenlaan 200B, 3001 Leuven, Belgium

e-mail: Marian.Lazar@wis.kuleuven.be

S. Poedts

e-mail: Stefaan.Poedts@ wis.kuleuven.be 
functions (VDFs) become skewed along the ambient magnetic field (Marsch et al., 1982; Pilipp et al., 1987; Salem et al., 2003; Stverak et al., 2008).

Recently, it has been shown that the solar-wind ions can develop strong pitch-angle anisotropies when the ions pass through the magnetohydrodynamic (MHD) termination shock (Fahr and Siewert, 2007). At large solar distances, mechanisms of adiabatic cooling (e.g., the conservation of the two magnetic CGL invariants) can, however, prevent the solar wind from developing strong pressure anisotropies (Fahr and Siewert, 2008). The acceleration processes in solar flares and wind are able to heat particles preferentially along the background magnetic field (Miller et al., 1997; Miller, 1998; Paesold and Benz, 1999; Fletcher and Hudson, 2008). Thus, in interplanetary plasmas the electrons and ions gain energy either through the cyclotron resonance and the transit time (Landau) damping of the linear waves (Fisk, 1976; Miller, 1991, 1997; Ma and Summers, 1998; Summers and Ma, 2000) or through nonlinear Landau damping, in the presence of large-amplitude waves and plasma turbulence (Miller, 1991; Leubner, 2000; Shizgal, 2007).

A substantial amount of kinetic energy residing in the direction of the magnetic field may drive either a Weibel-like instability of the ordinary mode propagating perpendicular to the magnetic field (Hamasaki, 1968a) or a firehose instability (FHI), which is able to excite and destabilize the circularly polarized Alfvén modes propagating along the magnetic field (Hollweg and Völk, 1970). The importance of these modes for the transport of energy and particles in space plasmas is already very well known (Gary, 1993; Marsch, 2006). For instance, recently, it has been demonstrated that the Weibel-like instabilities provide a plausible mechanism for the origin of the fluctuating solar-wind magnetic fields (Stockem, Lerche, and Schlickeiser, 2006).

The electron FHI is also expected to play an important role in solar flares and wind; see for instance the discussions in Paesold and Benz (1999) and Li and Habbal (2000). As the instability occurs, it constrains the increase of the electron-temperature anisotropy, thus explaining the observations. Simulations have confirmed that the generated magneticfield fluctuations scatter the electrons, reducing their anisotropy (Messmer, 2002; Gary and Nishimura, 2003; Paesold and Benz, 2003; Camporeale and Buragess, 2008). Thus, the electron FHI could be one of the most efficient mechanisms of temperature isotropization in solar flares, limiting electron anisotropy and thus providing the necessary condition for further acceleration (Messmer, 2002).

Both the Weibel and kinetic firehose instabilities are nonresonant with plasma particles, and both have their origin in the velocity anisotropy of plasma particles, but the physical mechanisms responsible for their generation are essentially different. The Weibel instability (WI) is a magnetic instability nonresonantly induced in a plasma with temperature anisotropy caused by bunching of the plasma particles by the Lorentz force of a magnetic perturbation, with bunches interacting and enhancing the perturbation. This instability has originally been described in the absence of an ambient magnetic field, either for an anisotropic plasma with a bi-Maxwellian distribution (Weibel, 1959) or for counterstreaming cold plasmas (Fried, 1959). In contrast to this, the FHI grows only in the presence of a stationary magnetic field, where an excess of parallel kinetic energy will push and bend the field lines, which, as a result, can amplify any (low-frequency) transverse perturbation propagating along the background magnetic field. Whereas the ordinary unstable mode propagates perpendicular to the regular magnetic field, the FHI develops along the magnetic field. Since the frequency of the FHI is in the range of the ion gyrofrequency (with the electrons being nonresonant) the ions are resonant and could gain energy, making the FHI responsible for the transfer of the electron energy to the ions (Paesold and Benz, 1999).

Here we prove that the WI of the ordinary mode is an important competitor for the FHI, developing, in general, much faster than the FHI. Consequently, the existence of the FHI 
will be limited to temperature anisotropies markedly smaller, within a well-defined interval provided here. These two instabilities have been extensively described in previous works (Paesold and Benz, 1999; Li and Habbal, 2000; Tautz and Schlickeiser, 2006; Lazar and Poedts, 2009), and therefore, here, in Section 2, we only present the most important results, which allow us to investigate their interplay. Thus, the maximum growth rates reached at saturation are evaluated for each instability. The maximum growth rate gives us the order of magnitude for the growth time of the instability: the larger the maximum growth rate is, the faster the instability is. The temperature anisotropy thresholds are then compared in Section 3, providing the existence condition for the FHI. These criteria are discussed in Section 4 for some particular cases in space plasmas, and our conclusions are finally given in the last section.

\section{Dispersion Formalism}

The considered plasma system is homogeneous, largely extended, and immersed in a uniform magnetic field $\left(\mathbf{B}_{0}\right)$. The unperturbed particle distributions are assumed to be anisotropic in a gyrotropic bi-Maxwellian form

$$
F_{a}=\frac{1}{\pi^{3 / 2} w_{\|, a} w_{\perp, a}^{2}} \exp \left(-\frac{v_{\|}^{2}}{w_{\|, a}^{2}}-\frac{v_{\perp}^{2}}{w_{\perp, a}^{2}}\right),
$$

where $v_{\|}$and $v_{\perp}$ are the usual velocity components parallel and perpendicular to $\mathbf{B}_{0}$, respectively, and $w_{\|, a}=\left(2 k_{\mathrm{B}} T_{\|, a} / m_{a}\right)^{1 / 2}$ and $w_{\perp}=\left(2 k_{\mathrm{B}} T_{\perp, a} / m_{a}\right)^{1 / 2}$ are the corresponding thermal velocities for each sort $a$ of plasma particles (for electrons $a=\mathrm{e}$, for ions $a=\mathrm{i}$, and for protons $a=\mathrm{p})$. The mass of ions is however sufficiently large $\left(m_{\mathrm{i}} \gg m_{\mathrm{e}}\right)$ and we will assume them to be rigid and isotropically distributed.

In a plasma that is sufficiently hot or rarified, binary collisions can be neglected and the dispersion analysis is based on the linearized Vlasov equation combined with Maxwell's equations.

\subsection{Weibel-like Unstable Ordinary Mode}

From the plasma modes propagating perpendicular to the magnetic field, the ordinary mode is the only one affected by the temperature anisotropy. The dispersion relation of the ordinary mode is usually given in terms of the Bessel function $J_{n}(z)$ of the first kind and order $n$ (Hamasaki, 1968a; Tautz and Schlickeiser, 2006),

$$
1-\frac{k_{\perp}^{2} c^{2}+\sum_{a} \omega_{\mathrm{p}, a}^{2}}{\omega^{2}}-\sum_{a} \frac{\omega_{\mathrm{p}, a}^{2}}{\omega^{2}} \sum_{n=-\infty}^{\infty} \int \mathrm{d}^{3} v \frac{J_{n}^{2}\left(z_{a}\right)}{1-\omega /\left(n \Omega_{a}\right)} \frac{v_{\|}^{2}}{v_{\perp}} \frac{\partial F_{a}}{\partial v_{\perp}}=0,
$$

with

$$
z_{a}=\frac{k_{\perp} v_{\perp}}{\Omega_{a}} .
$$

The polarization of this transverse mode is along the magnetic field, $\mathbf{E} \| \mathbf{B}_{0}$, and $\mathbf{k}_{\perp} \perp \mathbf{B}_{0}$. Here, $\omega$ and $k$ are, respectively, the frequency and the wave number of the plasma modes, $c$ is the speed of light in vacuum, $\Omega_{a}=q_{a} B_{0} /\left(m_{a} c\right)$ is the (nonrelativistic) gyrofrequency, and $\omega_{\mathrm{p}, a}=\left(4 \pi n_{a} e^{2} / m_{a}\right)^{1 / 2}$ is the plasma frequency for plasma particles of sort $a$. 
Inserting the distribution function (1) in Equation (2) and neglecting the slow reaction of ions, which only form a neutralizing background, the dispersion relation (2) becomes (Davidson and Wu, 1970; Tautz and Schlickeiser, 2006)

$$
\frac{\omega^{2}-k_{\perp}^{2} c^{2}}{\omega_{\mathrm{p}, \mathrm{e}}^{2}}=1-\frac{w_{\|, \mathrm{e}}^{2}}{w_{\perp, \mathrm{e}}^{2}}\left[1+\mathrm{e}^{-u_{\mathrm{e}}} \sum_{n=-\infty}^{\infty} \frac{I_{n}^{2}\left(u_{\mathrm{e}}\right)}{n^{2} \Omega_{\mathrm{e}}^{2} / \omega^{2}-1}\right],
$$

where

$$
u_{\mathrm{e}}=\frac{k_{\perp}^{2} w_{\perp, \mathrm{e}}^{2}}{2 \Omega_{\mathrm{e}}^{2}}
$$

and $I_{n}(u)$ denotes the modified Bessel function of the first kind. A further reduction of the infinite sum in the dispersion relation involves hypergeometric functions, which make the description of the instability less transparent. However, in our study here, we only need to estimate both the maximum growth rate reached at saturation and the threshold of the instability.

If we restrict ourselves, for example, to large temperature anisotropies so that $w_{\|, \mathrm{e}} \gg$ $w_{\perp, \mathrm{e}}$ and $u_{\mathrm{e}} \ll 1$, the dispersion relation (4) yields aperiodic solutions of the form (Hamasaki, 1968b; Lazar, Schlickeiser, and Poedts, 2009)

$$
\omega^{2}=-\frac{\omega_{\mathrm{p}, \mathrm{e}}^{2}}{k^{2} c^{2}+\omega_{\mathrm{p}, \mathrm{e}}^{2}+\Omega_{\mathrm{e}}^{2}}\left[\frac{1}{2} k^{2} w_{\|, \mathrm{e}}^{2}-\Omega_{\mathrm{e}}^{2}\left(\frac{k^{2} c^{2}}{\omega_{\mathrm{p}, \mathrm{e}}^{2}}+1\right)\right] .
$$

Note that in the presence of a static magnetic field, aperiodic solutions are found for wave numbers larger than the threshold value imposed by the electron gyrofrequency,

$$
k>k_{\mathrm{t}}=\Omega_{\mathrm{e}}\left(\frac{1}{2} w_{\|, \mathrm{e}}^{2}-c^{2} \frac{\Omega_{\mathrm{e}}^{2}}{\omega_{\mathrm{p}, \mathrm{e}}^{2}}\right)^{-1 / 2}=\frac{\sqrt{2} \Omega_{\mathrm{e}}}{w_{\|, \mathrm{e}}}\left(1-\frac{2}{\beta_{\|}}\right)^{-1 / 2},
$$

where the parallel plasma $\beta$ is given by $\beta_{\|}=8 \pi n k_{\mathrm{B}} T_{\|, \mathrm{e}} / B_{0}^{2}$. For nonmagnetized plasmas this threshold vanishes $\left[k_{\mathrm{t}}\left(\Omega_{\mathrm{e}}=0\right)=0\right]$. The instability can grow for a not so intense ambient magnetic field, lower than a critical value. This is in agreement with the recent derivations of Stockem, Lerche, and Schlickeiser (2006) for the Weibel instability in two cold counterstreaming plasmas. Here, the critical magnetic field depends on the parallel thermal velocity and is given by the square root in Equation (7),

$$
B_{0}<B_{0, c}=\sqrt{2 \pi n_{0} m_{\mathrm{e}}} w_{\|, \mathrm{e}} .
$$

The growth rate $\omega_{\mathrm{i}}=\Im(\omega)$ from Equation (6) admits a maximum expressed by

$$
\omega_{\mathrm{i}}^{\max }=\omega_{\mathrm{i}}(k \rightarrow \infty)=\omega_{\mathrm{p}, \mathrm{e}} \frac{w_{\|, \mathrm{e}}}{\sqrt{2} c}\left(1-\frac{2}{\beta_{\|}}\right)^{1 / 2} .
$$

For magnetic fields that are sufficiently weak, $\beta_{\|}^{-1} \rightarrow 0$, the growth rate can reach a maximum $\omega_{\mathrm{i}}^{\max } \approx \omega_{\mathrm{p}, \mathrm{e}}\left(w_{\|, \mathrm{e}} / \sqrt{2} c\right)=\omega_{\mathrm{p}, \mathrm{e}} / \mu_{\mathrm{e}}$, where, for nonrelativistic plasma temperatures, $\mu_{\mathrm{e}}=\sqrt{m_{\mathrm{e}} c^{2} /\left(k_{\mathrm{B}} T_{\|, \mathrm{e}}\right)}>10$. However, for a sufficiently large value of $\beta_{\|}$, the electron gyrofrequency is at least one order of magnitude less than the plasma frequency. It is therefore clear that, at saturation, this mode tends to have a wavelength of the order of the electron gyroradius and a growth rate of the order of the electron gyrofrequency. This is also confirmed by the exact numerical plots from Tautz and Schlickeiser (2006). 
The condition for the existence of this instability is given in Equation (8) that reads $\beta_{\|}>2$. This condition is derived in the limit of very large anisotropies by neglecting the transverse thermal spread, $w_{\perp, \mathrm{e}} \rightarrow 0$. For a finite $w_{\perp, \mathrm{e}} \neq 0$, the existence condition for the WI becomes more restrictive (Hamasaki, 1968a):

$$
\beta_{\|}>b_{1}=\frac{2}{1-\left(\frac{3}{2}\right)^{1 / 2} \frac{w_{\perp, \mathrm{e}}}{w_{\|, \mathrm{e}}}}>2
$$

\subsection{Firehose Instability}

The electromagnetic modes propagating along the ambient magnetic field (i.e., $\mathbf{k} \| \mathbf{B}_{0}$ ) are described by the following general dispersion relation (Tautz and Schlickeiser, 2005; Lazar and Poedts, 2009):

$$
\begin{aligned}
1 & -\frac{k^{2} c^{2}}{\omega^{2}}+\frac{\pi}{\omega^{2}} \sum_{a} \omega_{\mathrm{p}, a}^{2} \int_{-\infty}^{\infty} \frac{\mathrm{d} v_{\|}}{\omega-k v_{\|} \pm \Omega_{a}} \\
& \times \int_{0}^{\infty} \mathrm{d} v_{\perp} v_{\perp}^{2}\left[\left(\omega-k v_{\|}\right) \frac{\partial F_{a}}{\partial v_{\perp}}+k v_{\perp} \frac{\partial F_{a}}{\partial v_{\|}}\right]=0 .
\end{aligned}
$$

The electron firehose instability arises when the plasma is hotter along the stationary magnetic field (i.e., when $T_{\|}>T_{\perp}$ ). This instability is left-handed (LH) circularly polarized at $\mathbf{k} \times \mathbf{B}_{0}=0$, and the electrons are nonresonant. Only the ions are cyclotron resonant, and initially their unperturbed distribution function is assumed to be isotropic, $w_{\|, \mathrm{i}}=w_{\perp, \mathrm{i}}=$ $w_{\mathrm{i}}=\left(2 k_{\mathrm{B}} T / m_{\mathrm{i}}\right)$.

Inserting the distribution function (3) into the dispersion relation (11) gives

$$
1-\frac{k^{2} c^{2}}{\omega^{2}}+\frac{\omega_{\mathrm{p}, \mathrm{e}}^{2}}{\omega^{2}}\left\{\frac{\omega}{k w_{\|, \mathrm{e}}} Z\left(f_{\mathrm{e}}\right)-A_{\mathrm{e}}\left[1+f_{\mathrm{e}} Z\left(f_{\mathrm{e}}\right)\right]\right\}+\frac{\omega_{\mathrm{p}, \mathrm{i}}^{2}}{\omega^{2}} \frac{\omega}{k w_{\mathrm{i}}} Z\left(f_{\mathrm{i}}\right)=0,
$$

where $A_{\mathrm{e}}=1-w_{\perp, \mathrm{e}}^{2} / w_{\|, \mathrm{e}}^{2}$ and $Z(f)$ is the standard Fried and Conte (1961) plasma dispersion function with the argument

$$
f_{a}=\frac{\omega-\Omega_{a}}{k w_{\|, a}}
$$

The growth rates of the FHI can be obtained by solving Equation (12) exactly numerically, and some examples are plotted in Figure 1 for an electron - proton plasma with large temperature anisotropies and parameters estimated for the solar flares (Paesold and Benz, 1999). Such high temperatures are supported by the EUV and X-ray emissions, which are already a common characteristic signature for the presence of the energetic electrons $\left(k_{\mathrm{B}} T_{\mathrm{e}}>10 \mathrm{KeV} \approx 100 \mathrm{MK}\right)$ in impulsive solar flares (Miller et al., 1997), and by robust mechanisms of acceleration along the magnetic field lines: the transit-time damping of the fast modes (Miller, 1997; Lenters and Miller, 1998), or the Alfvén waves, which, in the presence of strong gradients, can generate field-aligned electric fields accelerating electrons to energies of $10 \mathrm{KeV}$ and above (Fletcher and Hudson, 2008). According to Figure 1, at saturation, the instability can reach a maximum growth rate of the order of the proton gyrofrequency, which is considerably smaller, namely $m_{\mathrm{p}} / m_{\mathrm{e}} \approx 2 \times 10^{3}$ times less than the electron gyrofrequency. 
Figure 1 The growth rates of the FHI for three temperature anisotropies $T_{\mathrm{e}, \|} / T_{\mathrm{e}, \perp}=12.5$ (long-dashed line), 13

(short-dashed line), and 14 (solid line). The electron and proton plasma parameters are $n_{\mathrm{e}}=5 \times 10^{10} \mathrm{~cm}^{-3}$, $T_{\mathrm{e}, \perp}=T_{\mathrm{p}, \perp}=T_{\mathrm{p}, \|}=10^{7} \mathrm{~K}$, and $B_{0}=100 \mathrm{G}$.

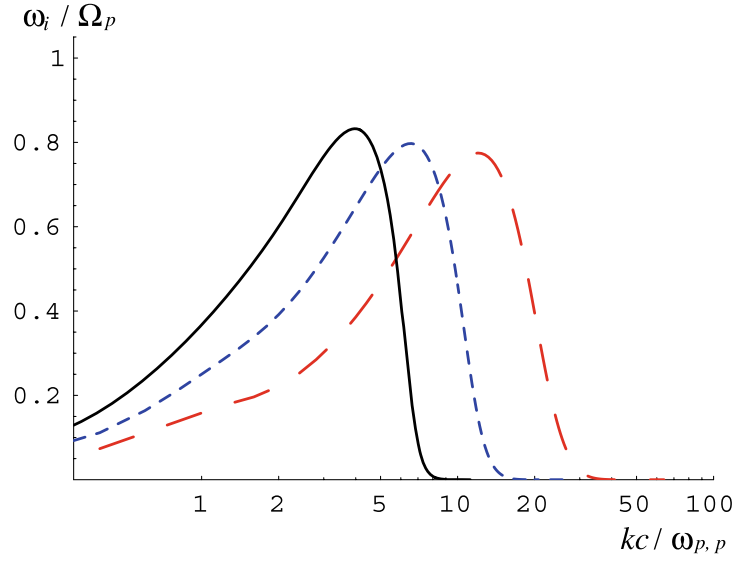

The electrons are nonresonant $\left(f_{\mathrm{e}} \gg 1\right)$, and the ions (protons) are resonant $\left(f_{\mathrm{i}}<1\right)$, and in Equation (12), we use power series of the plasma dispersion function (Fried and Conte, 1961) and obtain complex solutions $\omega=\omega_{\mathrm{r}}+\imath \omega_{\mathrm{i}}$ with the growth rate

$$
\frac{\omega_{\mathrm{i}}}{\Omega_{\mathrm{i}}}=\pi^{1 / 2} \frac{k^{2} c^{2}}{\omega_{\mathrm{p}, \mathrm{i}}^{2}}\left(\frac{A_{\mathrm{e}}}{2} \beta_{\|}-1\right)\left(\frac{k w_{\mathrm{i}}}{\Omega_{\mathrm{i}}}+\pi \frac{\Omega_{\mathrm{i}}}{k w_{\mathrm{i}}}\right)^{-1} .
$$

As one can see from Equation (14), the FHI is also suppressed by increasing the background magnetic field, and thus the firehose mode will be unstable, $\omega_{\mathrm{i}}>0$, only for a sufficiently large parallel plasma $\beta$ so that (Lazar and Poedts, 2009)

$$
\beta_{\|}>b_{2}=\frac{2}{1-\left(\frac{w_{\perp, \mathrm{e}}}{w_{\|, \mathrm{e}}}\right)^{2}}>2 .
$$

The criterion for the existence of the FHI is similar to that of the WI in Equation (10), but the thresholds are quite different $\left(b_{1} \neq b_{2}\right)$.

\section{WI versus FHI}

The estimations just made show that the WI growth rate is several orders of magnitude larger than that of the FHI. Thus, the first important conclusion here is that the WI grows much faster, relaxing the temperature anisotropy even before the FHI can develop. The existence of the FHI in a high- $\beta$ plasma will therefore be decided by conditions (10) and (15): Since $b_{2} \leqslant b_{1}$, the existence of the FHI is severely limited to a parallel plasma $\beta$ in the interval

$$
b_{2} \leqslant \beta_{\|} \leqslant b_{1} .
$$

In the absence of a thermal velocity spread perpendicular to $\mathbf{B}_{0}\left(w_{\perp, \mathrm{e}} \rightarrow 0\right)$ this interval vanishes, $b_{1}=b_{2}=2$, and the FHI cannot grow. Only the existence of a perpendicular thermal velocity spread will make the FHI more effective, but only in a narrow band, as is shown in Figures 2 and 3.

The existence condition (16) provides the range of $\beta_{\|}$where the temperature anisotropy $w_{\|, \mathrm{e}} / w_{\perp, \mathrm{e}}$ is given. Otherwise, for practical purposes, this condition can take different forms. 
Figure 2 Limits of the FHI as given by condition (16) in a diagram of $\beta_{\|}$versus $\beta_{\perp}$; the $\mathrm{FHI}$ is restricted to the colored area.

Figure 3 Limits of the FHI as given by condition (16) in a diagram of temperature anisotropy $T_{\|, \mathrm{e}} / T_{\perp, \mathrm{e}}$ versus perpendicular $\beta$

$\left(\beta_{\perp}=T_{\perp, \mathrm{e}} \beta_{\|} / T_{\|, \mathrm{e}}\right)$; the FHI is effective only in the colored area. For a given $\beta_{\perp}$ the FHI will relax the temperature anisotropies delimited by the dashed lines and laying in between the boundaries of the colored area. $\beta_{\|}$
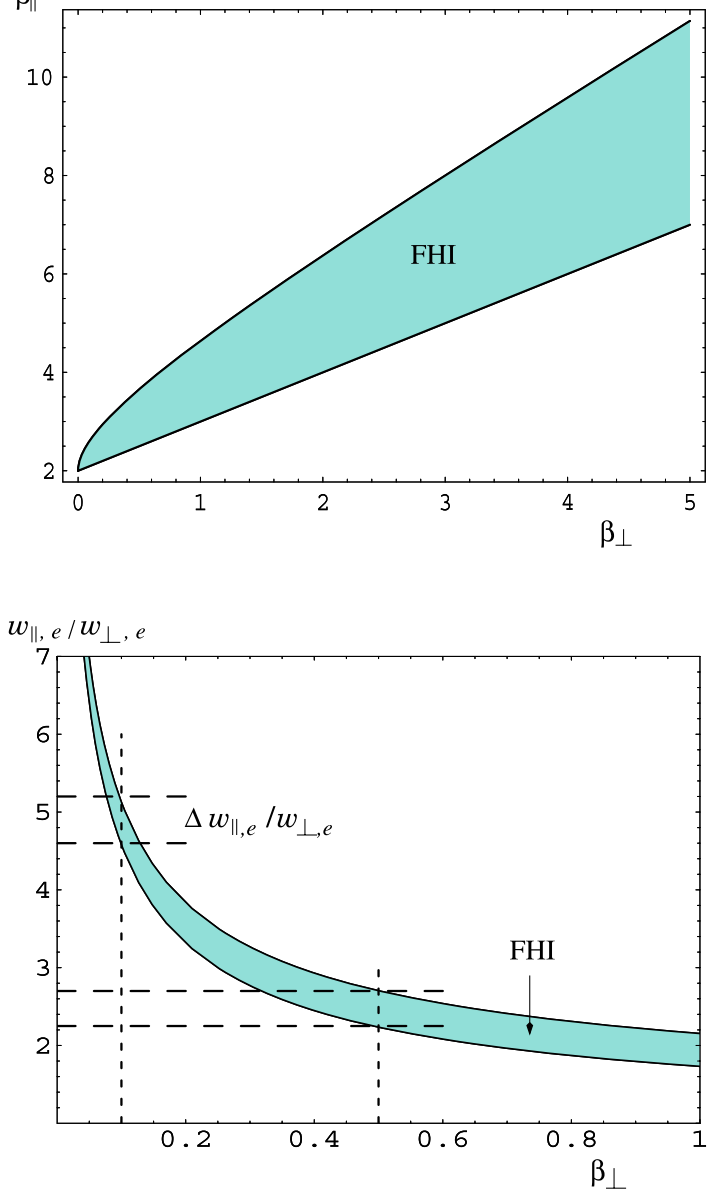

Thus, if $\beta_{\perp}$ is known, condition (16) will limit $\beta_{\|}$to

$$
\beta_{\perp}+2 \leqslant \beta_{\|} \leqslant \frac{\beta_{\perp}}{4}\left[\left(\frac{3}{2}\right)^{1 / 2}+\left(\frac{3}{2}+\frac{8}{\beta_{\perp}}\right)^{1 / 2}\right]^{2}
$$

or the temperature anisotropy to

$$
\left(\frac{2}{\beta_{\perp}}+1\right)^{1 / 2} \leqslant\left(\frac{T_{\|, \mathrm{e}}}{T_{\perp, \mathrm{e}}}\right)^{1 / 2}=\frac{w_{\|, \mathrm{e}}}{w_{\perp, \mathrm{e}}} \leqslant \frac{1}{2}\left[\left(\frac{3}{2}\right)^{1 / 2}+\left(\frac{3}{2}+\frac{8}{\beta_{\perp}}\right)^{1 / 2}\right] .
$$

In a diagram of $\beta_{\|}$versus $\beta_{\perp}$ displayed in Figure 2, condition (17) restricts the existence of the FHI to the colored intermediary area, where $\beta_{\|}>2$ is sufficiently large but limited by the threshold of the WI. Condition (18) is also displayed in Figure 3, by using a diagram of the temperature anisotropy $w_{\|, \mathrm{e}} / w_{\perp, \mathrm{e}}$ versus $\beta_{\perp}$, and the existence of the FHI is again limited to a narrow colored strip. Some applications can be imagined using these diagrams to determine the plasma parameters when the presence of the low-frequency Alfvén waves excited by the firehose instability can be confirmed by the observations. If the perpendicular 
$\beta$ is already estimated, the parallel $\beta$ or the temperature anisotropy that drives the FHI instability can be determined by using one of the diagrams in Figures 2 or 3 .

\section{Application to Solar Plasmas}

We now consider some particular cases of magnetized plasmas presently expected to be representative for the solar corona, solar flares, and the solar wind (the acceleration region of the outer corona). The parameters used to characterize such plasma systems are selected in Table 1, after Pallavicini, Serio, and Vaiana (1977) and Aschwanden (2004).

If we consider first the solar corona, the mean values for the plasma parameters are listed in the first column in Table 1, and one possible application here is quite simple. Common values of the plasma $\beta$ are expected to be very small in the corona, much less than unity, and therefore only the presence of a sufficiently large temperature anisotropy will guarantee the existence of the FHI. We consider, for example, $\beta_{\perp}=\beta_{\mathrm{e}}=0.1$ and then conditions (17) and (18) will provide narrow limits for the existence of the FHI as follows: $2.1 \leqslant \beta_{\|} \leqslant 2.62$ and $4.58 \leqslant w_{\|, \mathrm{e}} / w_{\perp, \mathrm{e}} \leqslant 5.11$, respectively.

The plasma $\beta$ for the solar wind in the acceleration region of the outer corona takes moderately large values. We choose as reference $\beta_{\perp}=\beta_{\mathrm{e}}=3$, which is given in Table 1 , and the inequalities (17) and (18) will provide broader limits for the FHI: $5 \leqslant \beta_{\|} \leqslant 8$, and $1.3 \leqslant w_{\|, \mathrm{e}} / w_{\perp, \mathrm{e}} \leqslant 1.63$.

The mean values estimated for plasma parameters in solar flares spread over some intervals, as shown in Table 1, and therefore simple calculation of plasma $\beta$ provides very small values (much less than unity), as well as very large values (up to 100). But such high values probably fail to explain the occurrence of solar flares unless the magnetic Reynolds number is sufficiently large and the magnetic field is frozen in the flow and moves with it. The case considered by Paesold and Benz (1999) could be suggestive and it is also reiterated by Lazar and Poedts (2009), namely, that of an electron - proton plasma with $n_{\mathrm{e}}=5 \times 10^{10} \mathrm{~cm}^{-3}$ and $T_{\mathrm{e}}=10^{7} \mathrm{~K}$, in a magnetic field $B_{0}=100 \mathrm{G}$, which yields $\beta_{\mathrm{e}} \simeq 0.2$. As in the previous works, if we take this value as reference $\beta_{\perp}=\beta_{\mathrm{e}}=0.2$, conditions (17) and (18) provide us with the following limits for the existence of the FHI: $2.2 \leqslant \beta_{\|} \leqslant 2.94$ and $3.32 \leqslant w_{\|, \mathrm{e}} / w_{\perp, \mathrm{e}} \leqslant 3.83$. This gives us a narrow interval for the temperature anisotropy, $11.02 \leqslant T_{\|, \mathrm{e}} / T_{\perp, \mathrm{e}} \leqslant 14.67$, so that the FHI will be able to relax only the temperature anisotropies in this interval. The FHI growth rates plotted in Figure 1 are obtained for temperature anisotropies in this interval. Such graphical solutions have been provided in previous works (Paesold and Benz, 1999; Lazar and Poedts, 2009) even for large temperature anisotropies beyond the limit of this interval, but those examples do not affect the physical effects presented there. The next investigations should however limit the efficiency of the FHI to the intervals provided here in (16), (17), and (18).

Table 1 Solar plasma parameters for the corona, solar flares, and the solar wind (in the acceleration region).

\begin{tabular}{lccc}
\hline System & Corona & Flares & Solar Wind \\
\hline$n_{\mathrm{e}}\left(\mathrm{cm}^{-3}\right)$ & $10^{9}$ & $10^{9}-10^{11}$ & $10^{7}$ \\
$T_{\mathrm{e}}(\mathrm{K})$ & $3 \times 10^{6}$ & $10^{6}-10^{7}$ & $10^{6}$ \\
$B_{0}(\mathrm{G})$ & 10 & $100-500$ & 0.1 \\
$\omega_{\mathrm{p}, \mathrm{e}}\left(\mathrm{s}^{-1}\right)$ & $2 \times 10^{9}$ & $(2-20) \times 10^{9}$ & $2 \times 10^{8}$ \\
$\Omega_{\mathrm{e}}\left(\mathrm{s}^{-1}\right)$ & $2 \times 10^{8}$ & $(2-9) \times 10^{9}$ & $2 \times 10^{6}$ \\
$\beta_{\mathrm{e}}$ & $\leqslant 0.2$ & $0.01-100$ & $\geqslant 3$ \\
\hline
\end{tabular}




\section{Conclusions}

We have described the interplay of the WI and the FHI, which are different, but they both are driven by a thermal-plasma anisotropy with an excess of kinetic energy in the direction of the ambient magnetic field. The growth rates reached at saturation are compared for each instability, and it is found that the WI is much faster, developing well before the growth of the FHI.

These two instabilities are inhibited by the presence of an ambient magnetic field, namely, the interplanetary magnetic field in space plasmas. This makes the instability effective only for a temperature anisotropy larger than a threshold value that depends on the ambient magnetic field. Here we have shown that the WI admits an anisotropy threshold larger than that of the FHI. This is very important because it gives the FHI a chance to grow because the anisotropy does not cross the threshold of the WI. The exact boundary conditions for the existence of the FHI are provided in the inequalities (16), (17), and (18), in terms of the plasma betas, both parallel and perpendicular, and the temperature anisotropy. In the previous section, these limits have been calculated explicitly for three distinct regions in the solar space plasma: the corona, solar flares, and the solar wind in the acceleration region.

The low-frequency spectrum of Alfvén waves can be excited in space plasmas by the FHI instability. If such signals are detected, and this seems to be presently possible with highresolution observations using, for example, the Coronal Multi-Channel Polarimeter (CoMP) for detecting the Alfvénic motions in the corona (Tomczyk et al., 2007) or the Hinode X-ray Telescope for solar flares and jets (Cirtain et al., 2007), then we can use conditions (16), (17), and (18) or the diagram in Figures 2 and 3 to determine some of the plasma parameters. Since the colored band in Figure 3 is narrow, for a known perpendicular $\beta$, the temperature anisotropy can be determined with a sufficiently good accuracy.

Acknowledgements The authors acknowledge financial support from the Research Foundation Flanders FWO Belgium. These results were obtained in the framework of the projects GOA/2009-009 (K.U. Leuven), G.0304.07 (FWO - Vlaanderen), and C 90347 (ESA Prodex 9). Financial support by the European Commission through the SOLAIRE Network (MTRN-CT-2006-035484) is gratefully acknowledged. The numerical results were obtained on the HPC cluster VIC of the K.U. Leuven. We thank the anonymous referee for constructive comments.

\section{References}

Aschwanden, M.J.: 2004, Physics of the Solar Corona, Springer, New York.

Camporeale, E., Burgess, D.: 2008, J. Geophys. Res. 113, A07107.

Cirtain, J.W., Golub, L., Lundquist, L., van Ballegooijen, A., Savcheva, A., Shimojo, M., et al.: 2007, Science 318, 1580.

Davidson, R.C., Wu, C.S.: 1970, Phys. Fluids 13, 1407.

Fahr, H.-J., Siewert, M.: 2007, Astrophys. Space Sci. Trans. 3, 21.

Fahr, H.-J., Siewert, M.: 2008, Astron. Astrophys. 484, L1.

Fisk, L.A.: 1976, J. Geophys. Res. 81, 4633.

Fletcher, L., Hudson, H.S.: 2008, Astrophys. J. 675, 1645.

Fried, B.D.: 1959, Phys. Fluids 2, 337.

Fried, B.D., Conte, S.D.: 1961, The Plasma Dispersion Function, Academic Press, New York.

Gary, S.P.: 1993, Theory of Space Plasma Microinstabilities, University Press, Cambridge.

Gary, S.P., Nishimura, K.: 2003, Phys. Plasmas 10, 3571.

Hamasaki, S.: 1968a, Phys. Fluids 11, 2724.

Hamasaki, S.: 1968b, Phys. Fluids 11, 1174.

Hollweg, J.V., Völk, H.J.: 1970, J. Geophys. Res. 75, 5297.

Lazar, M., Poedts, S.: 2009, Astron. Astrophys. 494, 311. 
Lazar, M., Schlickeiser, R., Poedts, S.: 2009, Phys. Plasmas, in preparation.

Lenters, G.T., Miller, J.A.: 1998, Astrophys. J. 493, 451.

Leubner, M.P.: 2000, Planet. Space Sci. 48, 133.

Li, X., Habbal, S.R.: 2000, J. Geophys. Res. 105, 27377.

Ma, C., Summers, D.: 1998, Geophys. Res. Lett. 25, 4099.

Marsch, E.: 2006, Living Rev. Solar Phys. 3, http://www.livingreviews.org/lrsp-2006-1.

Marsch, E., Muhlhauser, K.H., Schwenn, R., Rosenbauer, H., Pilipp, W.G., Neubauer, F.M.: 1982, J. Geophys. Res. 87, 52.

Messmer, P.: 2002, Astron. Astrophys. 382, 301.

Miller, J.A.: 1991, Astrophys. J. 376, 342.

Miller, J.A.: 1997, Astrophys. J. 491, 939.

Miller, J.A.: 1998, Space Sci. Rev. 86, 79.

Miller, J.A., Cargill, P.J., Emslie, A.G., Holman, G.D., Dennis, B.R., LaRosa, T.N., et al.: 1997, J. Geophys. Res. 102, 14631.

Paesold, G., Benz, A.O.: 1999, Astron. Astrophys. 351, 741.

Paesold, G., Benz, A.O.: 2003, Astron. Astrophys. 401, 711.

Pallavicini, R., Serio, S., Vaiana, G.S.: 1977, Astrophys. J. 216, 108.

Pilipp, W.G., Miggenrieder, H., Montgomery, M.D., Muhlhauser, K.H., Rosenbauer, H., Schwenn, R.: 1987, J. Geophys. Res. 92, 1075.

Salem, C., Hubert, D., Lacombe, C., Bale, S.D., Mangeney, A., Larson, D.E., Lin, R.P.: 2003, Astrophys. J. $\mathbf{5 8 5}, 1147$.

Shizgal, B.D.: 2007, Astrophys. Space Sci. 312, 227.

Stockem, A., Lerche, I., Schlickeiser, R.: 2006, Astrophys. J. 651, 584.

Stverak, S., Travnicek, P., Maksimovic, M., Marsch, E., Fazakerley, A.N., Scime, E.E.: 2008, J. Geophys. Res. 113, A03103.

Summers, D., Ma, C.: 2000, J. Geophys. Res. 105, 15887.

Tautz, R.C., Schlickeiser, R.: 2005, Phys. Plasmas 12, 122901.

Tautz, R.C., Schlickeiser, R.: 2006, Phys. Plasmas 13, 062901.

Tomczyk, S., McIntosh, S.W., Keil, S.L., Judge, P.G., Schad, T., Seeley, D.H., Edmondson, J.: 2007, Science 317, 1192.

Weibel, E.S.: 1959, Phys. Rev. Lett. 2, 83. 